INGENIERÍA INDUSTRIAL

\title{
Recursos y capacidades que inciden en el desempeño de los subsectores económicos colombianos 2003-2004: un acercamiento desde la teoría basada en recursos
}

INDUSTRIAL ENGINEERING

\section{Resources and capabilities that influence the performance of Colombian economic subsectors 2003- 2004: an approach from the theory based on resources}

\author{
Diana M. Escandon*, Alberto Arias*, Jairo A. Salas* § \\ Facultad de Ciencias Económicas y Administrativas. Pontificia Universidad Javeriana Cali* \\ §jasalas@javerianacali.edu.co,dmescandon@javerianacali.edu.co, \\ aarias@javerianacali.edu.co,
}

(Recibido: Agosto 08 de 2011 - Aceptado: Noviembre 19 de 2012)

\begin{abstract}
Resumen
Este artículo logra establecer como los recursos y capacidades, desarrollados por un conjunto de empresas agrupadas por subsectores económicos, le otorgan eficiencia operativa o diferenciación a un subsector frente a otro. En el estudio se utilizó un análisis factorial para determinar las variables independientes y luego, una Regresión Lineal Ponderada, para explicar el desempeño de los subsectores. Entre los principales hallazgos, se encuentra que el recurso humano, en términos de inversión en educación y capacitación y el desarrollo de recursos y capacidades tales como el capital humano destinado al manejo ambiental, tienen una incidencia positiva sobre la productividad laboral y por ende en la competitividad de los subsectores colombianos. Sin embargo, en el caso de la innovación, la inversión en $\mathrm{I}+\mathrm{D}$, la protección y divulgación de los procesos de innovación, reporta impactos negativos sobre la productividad, debido a la existencia predominante de innovaciones de tipo incremental. En conclusión, el aporte de este trabajo gira en torno a como los recursos y capacidades existentes en un subsector, pueden influir en las decisiones que tomen las empresas para mejorar su desempeño e impulsar a su vez la productividad de su subsector.
\end{abstract}

Palabras clave: Competitividad, Innovación, Recurso Humano, Recursos y capacidades

\begin{abstract}
The purpose of this article is to establish how resources and capabilities developed by a set of companies, grouped by economic sub-sectors, can bestow operating efficiency or differentiation on one sub-sector compared with another. This study used factor analysis to determine the independent variables, and then a weighted linear regression to explain the performance of the sub-sector. Among the key findings is that human resources, in terms of investment in education and training, and the development of resources and capabilities such as human capital, have a positive impact on labor productivity and thus on the competitiveness of Colombian sub-sectors. However, in the case of innovation, R \& D investment, protection and dissemination of innovation processes, reported negative impacts on productivity, due to the existence of different types of incremental innovations. In conclusion, the contribution of this work is focused on how the resources and capabilities in a subsector can influence the decisions made by companies to improve their performance and boost productivity of their subsectors.
\end{abstract}

Key Words: Competitiveness, Innovation, Human Resources, Resources and Capabilities 


\section{Introducción}

Porter y McGahan (1997) comprobaron que la rentabilidad de las empresas depende en casi $48 \%$ del esfuerzo individual que estas hacen para diseñar e implementar buenas estrategias competitivas, en un $18 \%$ del sector industrial donde se ubican y en un $30 \%$ del negocio o nicho específico en que compiten.

Es así como la productividad de las empresas, depende no solo de sus estrategias sino del sector industrial donde deciden competir. Es decir, que la estructura de la industria será un predictor de la rentabilidad futura que la empresa obtenga, y esto dependerá de si hay muchos o pocos competidores avivando la intensidad competitividad, de cómo sea el poder de negociación de los proveedores y clientes o de la existencia de amenazas de sustitutos o nuevos entrantes. Lo cierto es que las empresas reaccionan a todo esto obteniendo o generando recursos y capacidades que las lleven a mitigar estas fuerzas de la competencia o colocarlas a su favor.

Así habrá subsectores económicos más competitivos que otros, es decir, donde la productividad sea más alta. En este sentido, esta investigación busca explicar como los recursos y capacidades, desarrollados por un conjunto de empresas pertenecientes a un subsector, hacen que este tenga mayor productividad frente a otro dentro una misma economía, por tanto que las empresas existentes o que se creen en dicho subsector puedan apropiarse rápidamente de estas externalidades.

En la literatura científica existen gran cantidad de investigaciones sobre el éxito empresarial desde la óptica de la teoría basada en recursos y capacidades, entre ellas el capital humano, la innovación y la gestión ambiental.

La teoría de la firma basada en recursos y capacidades y la literatura sobre aprendizaje organizacional, afirman que la inversión en aprendizaje le permite a las organizaciones aumentar su base de conocimiento y de cualificación de sus recursos humanos (o competencias esenciales), así como su habilidad de asimilar y usar informaciones futuras (capacidad de absorción). Adicionalmente, la distribución heterogénea entre competencias esenciales y la capacidad de absorción es lo que le permite a la empresa obtener ventajas competitivas sustentables. (Cohen y Levinthal 1990; Hasenclever y Cassiolato 1998)

Existen diferentes postulados acerca de la contribución del recurso humano para la consecución de ventaja competitiva. Un primer grupo de autores, analizan el recurso humano por su contribución al proceso productivo por medio de aplicación de nuevos conocimientos a la organización. Este aspecto hace necesario que los empresarios generen políticas constantes de capacitación y actualización de conocimientos y de estabilidad del personal, que permitirán mejorar e interiorizar conocimientos y habilidades necesarios para lograr o aumentar la productividad empresarial; es decir se busca potencializar los recursos y capacidades de la empresa por medio de la capacitación o inversión en el capital humano para que le permita la consecución de una ventaja sobre sus competidores y que le aseguren su mantenimiento en el mercado. (Aguilar, 2000; Sastre y Aguilar 2000; Casanova 2002; Fernández, 2003).

Dentro de este mismo grupo podemos encontrar a Mertens (2002); Hasenclever y Cassiolato (1998) quienes afirman que la real contribución de los recursos humanos es cuando logran desarrollar capacidades que permitan la creación o el desarrollo de otros recursos, lo cual puede ser comprobado por medio del nivel de innovación que se logre incorporar a los procesos o productos finales de la organización. En consecuencia, se puede ver al recurso humano como elemento integrador y generador de habilidades para la utilización eficiente de los demás componentes de la ventaja competitiva. (Snell y Dean, 1992; Sisson y Store, 2002).

Por tanto, existe una relación directa entre la acumulación de capital humano, medida a partir del incremento de los niveles educativos, con la difusión tecnológica y la capacidad de adopción de innovaciones; es decir, los individuos más educados están en mejores condiciones de resolver problemas e incentivan más la innovación, haciendo que mejore el nivel de 
eficiencia y de consecución de las ganancias de la organización (Gill, 1989; Nelson y Phelps, 1996)

Por otra parte, encontramos un grupo de autores que plantea que la medición del recurso humano debe enfocarse en aspectos tales como la experiencia, poder de asimilación de conocimiento y la capacidad creadora, que puede ir desarrollando cada trabajador a medida que pasa el tiempo, es decir debe gestionarse el recurso humano (reclutamiento, selección, evaluación de resultados, formación y remuneración, así como políticas que enmarcan comportamientos y practicas dentro de las organizaciones) en pro del cumplimiento de las metas organizacionales. (Diéguez et al., 2007). Otros estudios, ratifican la relación entre el capital humano y la productividad, pero lo analizan desde los resultados, que se logra cuando se realizan inversiones acopladas a las necesidades reales de la organización y dirigidas a los individuos que puedan mejor aplicarla o utilizarla, es decir, debe existir un plan de desarrollo del capital humano (atracción, desarrollo y retención) al interior de cada organización que logre impulsarlo como activo inimitable, debido a la particularidad de necesidades de cada organización . (Koch y McGrath, 1996; Bartmess y Cerny, 1993; Hall, 1992).

Otros estudios encuentran que el impacto capital humano trasciende la empresa y se traslapa al sector (Nelson y Winter, 1992; Levitt y March, 1988), región o localidad en donde opera, a través de la difusión de conocimientos, interacción o cooperación empresarial y movilidad de los trabajadores (Cooke, Gómez, y Etxebarria, 1997; Howells, 1999; Carlsson, Jacobsson y otros, 2002; Iammarino, 2005). Estos factores solo pueden ser analizados mientras la interacción entre las empresas pueda ser de tipo frecuente o de cercanía geográfica, asegurando la verdadera transferencia de conocimiento (Iammarino, 2005; Cantwell y Molero, 2003; Malmberg, Sölvell y Zander, 1996).

Con base en lo anterior, se postula la primera hipótesis, donde el recurso humano, en términos de inversión en educación y capacitación, tendrá una relación positiva con la productividad laboral de un subsector (industria) en una economía determinada.

Otra variable que influye en el desempeño de una industria es la innovación, pues esta conduce a la generación de activos estratégicos por medio de la combinación de diferentes recursos que permitan soportar una diferenciación con respecto a las demás.

La innovación puede verse como un componente de información, conocimiento, esfuerzos en investigación y desarrollo, instituciones de colaboración, experiencia, habilidades y relaciones que, en conjunto con los recursos mencionados, conforman un cuerpo de capacidades que permite la consecución de aprendizaje organizacional ( Learning by doing; learning by changing; learning by using; learning by hiring y learning by training; learning by rearching y learning by $\mathrm{I}+\mathrm{D}$; learning by collaborating). Cada uno de los tipos de aprendizaje organizacional permite la creación de diferentes capacidades y por ende respuestas a requerimientos competitivos. (Teece y Pizano, 1994; Pitt, 2000; Robledo y Ceballos, 2008; Cohen y Levinthal, 1989; Paunero; 2003)

Algunos estudios establecen que la creación de innovación dentro de una organización y que permita obtener una ventaja competitiva, puede verse por medio de la consecución de capacidades estratégicas relacionadas con tecnologías y uso de recursos, capacidad de organización (coordinación de los recursos), capacidad de implementación u operativa (hacer las cosas bien), capacidad de adaptación al cambio o aprendizaje, capacidad de generación de incentivos o motivación. Estas capacidades permiten dar a las organizaciones, en términos de Barney (1991) una ambigüedad causal o según Grant (1991) una replicabilidad imperfecta, y así obtener o mantener la ventaja competitiva.

Estudios empíricos afirman que la innovación tiene un impacto positivo en la competitividad, especialmente cuando se desarrolla capacidades de relación o de participación en redes de cooperación, recursos humanos calificados 
e involucrados en procesos de aprendizaje continuo para poder realizar innovaciones constantes y habituales (Rodeiro y López, 2007). Por tanto, el no desarrollo de las capacidades mencionadas pueden llevar a que los procesos de innovación refleje efectos negativos en la generación de valor agregado, principalmente por la baja integración de las empresas al sistema científico tecnológico y, por la dificultad para transmitir el conocimiento adquirido o incorporarlo al proceso productivo (Espinosa, 2004; Martínez, 2001).

Por otra parte, el impacto de la innovación también puede depender de la disponibilidad de recursos financieros y por ende su aplicación se ajusta al análisis de la relación costo-beneficio, las expectativas de retorno de la inversión con respecto a otras que ofrezca el mercado, valor presente neto, entre otros. (Castellanos, Gálvez y Otros, 2006; Dosi, 1988; Uribe, 2007).

Otra posición con respecto a los efectos de la innovación, es que la asignación de recursos en este, hace disminuir la rentabilidad inmediata, pero su no implementación, podría dejar a una organización o hasta una industria por fuera del mercado, es decir "las empresas pueden quebrar si gastan demasiado en $\mathrm{I}+\mathrm{D}$, pero pueden desaparecer también si gastan demasiado poco". Matthews citado por Escorza (2001).

Por tanto, la innovación no se supedita al interés de una organización para su supervivencia sino al desarrollo y consolidación de un sistema de innovación social que debe ser soportado por recursos institucionales que permita la interacción de diferentes actores sociales (universidades, centros de desarrollo tecnológico, actores financieros, entre otros), permitiendo la creación y/o desarrollo de la competitividad de un sector, región o país. (Bell y Pavitt, 1992; Edquist, 1998; Freeman, 1998).

Otros estudios concluyen que la innovación no solo permite la creación de ventaja competitiva sino lograr su sostenibilidad, debido a que logra desarrollar competencias de tipo dinámico dentro de la organización. Estas competencias son de gran importancia, pues genera desarrollo constante de capacidades directivas y por ende dificulta descifrarla por parte de los competidores, las combinaciones de las habilidades organizativas, funcionales y tecnológicas, así como los conocimientos suscitados en el área de I $+\mathrm{D}$, desarrollo de productos, procesos, transferencia tecnológica, propiedad intelectual y en general el aprendizaje organizativo (Teece, 1982; Galán, 1998; Díaz, 2003; Fernández, 2003).

Esta situación hace que las organizaciones de gran tamaño tengan mayores dificultades en mantener o renovar las capacidades adquiridas para el proceso de creación de valor (Erber y Gersten, 1997). Sin embargo, las empresas pequeñas tienen dificultades debido a la carencia de recursos suficientes para hacer frente a los cambios del entorno y el logro de la ventaja competitiva. (Erber y Gersten, 1997; Fernández, 2003).

De lo anterior, se desprende la segunda hipótesis, donde se afirma que la innovación de un subsector (industria) en una economía determinada, desde la inversión en $\mathrm{I}+\mathrm{D}$, protección y divulgación de los procesos de innovación, permite aumentar la productividad laboral y por ende la competitividad.

Para algunos autores, es evidente que la existencia de regulaciones ambientales dentro de los diferentes sectores pueden hacer que se desarrollen nuevas capacidades y se utilicen de forma más eficiente los recursos organizacionales, esto debido a que se generan nuevos métodos de producción que tienden a ser innovadores y por consiguiente se ven reflejados en mayores niveles de competitividad, que se puede expresar en términos de Ecoeficiencia; es decir, una mejor calidad ambiental (uso de los recursos naturales, reciclaje de insumos y materiales adecuados), en una distribución eficiente de los recursos en el mercado, rendimientos financieros, minimización de riesgos financieros por la vía de reducción de riesgos ambientales, menores costos de capital y mejores controles de calidad, en el marco ambiental (Porter, 1995; Williams, 2002; Puig y Freire, 2007; Cruz A. 2000).

Por otra parte, otros autores afirman que la protección ambiental y su implementación no es que generen mejor utilización de los 
recursos ni desarrollo de capacidades, sino que conlleva a aumentar la necesidad de personal y en adoptar nuevas tecnologías, que al menos en el corto plazo, puede incidir negativamente en la competitividad, debido a que estos son costos directos de adopción y repercuten en los niveles de producción, inversión en capital y en el aumento de los costos de transacción, limitando al sector a responder a las exigencias del mercado, especialmente sus clientes. (Schmalensee, 1994; Puig y Freire, 2007; Duque y Tipia, 2007).

Por tanto, los efectos pueden ser tanto positivos como negativos, dependiendo de la posibilidad de desarrollar o mejorar capacidades y recursos para el desarrollo ambiental, en los diferentes sectores, que permitan nuevas formas de obtener resultados superiores y crear ventajas competitivas. Esto puede hacer que las empresas que se encuentren en ambientes más competitivos, adopten fácilmente nuevas prácticas que le permitirán desarrollan rápidamente capacidades para asumir políticas ambientales y absorber sus costos asociados, manteniendo su nivel de competitividad. (Duque y Tipia, 2007).

El comportamiento ambiental al interior de las organizaciones con respecto al manejo ambiental no es generalizado ni siquiera en países desarrollados. En el caso de la comunidad europea, algunas empresas asumen que la responsabilidad ambiental puede ser incorporada en la estrategia empresarial al ser comprobado la viabilidad técnico-económica y de mercadeo, es decir, el cambio en el comportamiento empresarial con respecto al manejo ambiental, tiene como factores determinantes asociados al logro a limitaciones de recursos financieros, humanos, tecnológicos y organizacionales (Taylor, 1993; Parizotto, 1995; Geisse, 1990).

Otro aspecto analizado en la literatura, es que los sectores que tienen mayores niveles de recursos y capacidades, en términos ambientales, pueden impactar por medio de la generación de redes de cooperación y de financiamiento, tanto de tipo sectorial, regional o inclusive global, logrando el desarrollo sostenible que es competencia de todos los actores sociales y al mismo tiempo fortaleciendo la capacidad técnica y política que le permita adaptarse a las nuevas exigencias sociales en términos ambientales. (Rodríguez, 2007; Millán y Nieto, 2003; Flórez y Jiménez, 2007; Puig y Freire, 2007).

De igual forma, el tamaño del área, el número y el nivel de formación de los empleados vinculados a la gestión ambiental, denotan los esfuerzos e inversiones en términos de recursos y capacidades para hacer frente a los retos y las limitaciones de las capacidades institucionales en este campo. Esto demuestra como la competitividad se puede lograr por medio del desarrollo de recursos y capacidades, especialmente de tipo humano capacitado en términos ambientales, que permite la creación de un diferencial en el mercado. (Rodríguez, 2007; Millán y Nieto, 2003).

Con base en lo expuesto se postula la tercera hipótesis, donde se afirma que el desarrollo de recursos y capacidades desde el capital humano en el manejo ambiental al interior de un subsector (industria) en una economía determinada, permite un efecto positivo en los niveles de productividad.

\section{Metodología}

\section{Muestra}

La muestra utilizada en este estudio es extraída de la encuesta Innovación y Desarrollo Tecnológico en la Industria Manufacturera 2003 y 2004 de Colombia. Los individuos a estudiar son 66 de los 73 subsectores (industrias) económicos colombianos según la clasificación CIIU, agrupados a 3 dígitos del CIIU.

Dicha encuesta fue realizada en conjunto por el Departamento Administrativo Nacional de Estadística (DANE), Departamento Nacional de Planeación (DNP), el Instituto Colombiano para el Desarrollo de la Ciencia y Tecnología "Francisco José de Caldas" (COLCIENCIAS).

\section{Descripción de la muestra}

De las 6145 empresas de la industria manufacturera que se tomaron como base para este estudio, se puede decir que $11,4 \%$ de ellas pertenecen al subsector de fabricación 
de prendas de vestir, excepto prendas de piel (código CIIU 181), el 6,8\% a fabricación de productos de plástico (código CIIU 252), el $6,2 \%$ a fabricación de otros productos químicos (código CIIU 242) y el 6,3\% a elaboración de productos de panadería, macarrones, fideos, alcuzcuz y productos farináceos similares (código CIIU 155).

Los subsectores que se destacan por lograr los mayores niveles de valor agregado son: con un 13,3\%, la fabricación de productos de la refinación del petróleo (código CIIU 232), seguido con un $9,1 \%$ la fabricación de otros productos químicos (código CIIU 242), con el $5,2 \%$ la fabricación de metales (código CIIU 273) y con el 4.8\%, la industrias básicas de metales preciosos y de metales no ferrosos (código CIIU 272).

El $60 \%$ de las empresas son pequeñas, es decir tiene entre 10 y 50 trabajadores o con activos totales entre 0,13 millones y menos de 1.3 millones de dólares. La mediana empresa representa el $28 \%$ del total de empresas encuestadas y solo el $12 \%$ se clasifica como gran empresa, debido a que cuentan con más de 200 trabajadores o tienen un total de activos superiores a 3,9 millones de dólares. Esto denota que la muestra está sesgada hacia las empresas más grandes, es decir, aquellas que movilizan la mayor proporción del valor agregado nacional.

Los trabajadores de estas empresas en un $53 \%$ son trabajadores que cuentan como nivel máximo de escolaridad el bachillerato, el $17 \%$ tienen la primaria finalizada, el $15 \%$ son profesionales, el $2 \%$ tienen postgrado y $0,05 \%$ son doctores.

En términos de Innovación, más del 50\% de las empresas se catalogan como no innovadoras y solo el $10 \%$ logran clasificarse como innovadoras en el sentido estricto.

\section{Variables del modelo}

Tomando como referente las variables incluidas en trabajos revisados en el marco conceptual y teniendo como referencia la información incluida en la base de datos, a continuación se establecieran las variables a utilizar en este trabajo.

\section{Variable Dependiente}

En este estudio se incluye como variable dependiente la productividad laboral (ValorAgregado). Esta variable muestra la contribución de valor agregado por cada trabajador. Este valor agregado es el resultado de la diferencia entre la producción y el consumo intermedio de cada subsector.

\section{Variables independientes.}

Por la cantidad de variables existentes dentro de encuesta que miden un mismo concepto, se decide realizar un análisis factorial como método de reducción de datos evitando inconvenientes de colinealidad entre las variables utilizadas en el análisis.

\section{Variables incluidas en los componentes para análisis factorial}

A continuación se agrupan las variables por dos componentes para realizar el análisis factorial:

Componente de Investigación y desarrollo

1. Empresas adecuadas tecnológicamente (empadectecno): Esta medida en términos del nivel de inversión destinada a la innovación

2. Empresas con innovación organizacional (empresasorganiz): Esta medida en términos del nivel de inversión destinada a la innovación

3. Empresas Incrementales: (empincrem)Esta medida en términos del nivel de inversión destinada a la innovación

4. Instrumentos para la Competitividad y el Desarrollo Productivo (intcompydesarrollo empresas que la usaron): Es medido en términos de empresas que utilizaron los instrumentos para la Competitividad y el Desarrollo productivo.

5. Instrumentos de Educación nacional, formación profesional y para el trabajo (insteducnalyprof Empresas que lo usaron): Es medido en términos de empresas que utilizaron los instrumentos de Educación nacional, formación profesional y para el trabajo. 
6. Cooperación de Empresas grupo (PropiosEmpresas que usan esta fuente): Empresas que utilizaron como fuente de cooperación empresas no pertenecientes a su grupo empresarial

7. Registros y aprobación de patentes (Registrospatente): Numero de patentes que se registran y logran ser aprobadas.

\section{Componente de Inversión en capital humano}

1. Postgrado: Muestra la cantidad de personal con nivel de postgrado

2. Universitario: Muestra la cantidad de personal con nivel universitario

3. Doctorado: Muestra la cantidad de personal con nivel de doctorado

4. Inversión en Capital humano: Monto en promedio que se invierte en capacitación del capital humano

5. Educación Intermedia: Muestra la cantidad de personal con nivel de educación intermedia (Técnico y tecnológico)

\section{Variable Ambiental}

1. Ambiental y manejo de recursos: Numero de personas en promedio vinculadas en cada empresa en la parte ambiental y el manejo de los residuos

\section{Métodos Estadísticos}

Como se dijo anteriormente se utiliza un análisis factorial para determinar las variables independientes pues la encuesta mide un mismo concepto con varias variables, dado que este es un método de reducción de datos y busca extraer de un grupo de variables un conjunto de factores que explican la covariancia entre dichas variables, es decir, este método logra crear un grupo de componentes, que resultan del análisis de sus varianzas, y de los cuales se puede crear el análisis de regresión sin que se presente problemas de colinealidad entre variables que expliquen lo mismo en una variable dependiente. Lo anterior se expresa de la siguiente manera: $\chi 1, \chi 2, \chi 3 \ldots$. Grupo de variables aleatorias que se explican por un grupo de factores comunes

F1 ........Fm (siendo $\mathcal{F}<\mho)$ y $n$ factores únicos u1.....un

$\chi 1=a 11 \mathcal{F} 1+\ldots \ldots+a 1 m \mathcal{F} m+d 1 u 1$

$\chi 2=a 21 \mathcal{F} 1+\ldots \ldots .+a 2 m \mathcal{F} m+d 2 u 2$

$\chi n=a n 1 \mathcal{F} 1+\ldots \ldots .+a 1 m \mathcal{F} m+d n u n$

En donde:

a11...... a $1 \mathrm{~m}$ Son los pesos factoriales de los factores comunes

$\mathcal{F} 1$............ $\mathcal{F} m$ Existen m Factores comunes tales que $\mathcal{F}<\mho$ (grado de saturación de cada factor)

$d n$ Es el peso factorial del factor único $u$ les el factor único.

En este modelo se pueden plantear los siguientes supuestos:

- Factores comunes y los únicos están mutuamente incorrelacionados (factores independientes).

- Los $\mathrm{m}$ factores son en número menor que las $\mathrm{n}$ variables, pues se desea pode generar una reducción de datos para explicar todas las variables.

Una vez establecidas todas las variables independientes, se utilizó una Regresión Lineal Ponderada, para explicar el desempeño de los subsectores. Este es un método de análisis que se usa cuando se cuenta con una variable dependiente que se supone que se encuentra relacionada con una o más variables independientes, y que permite obtener un modelo de mínimos cuadrados ponderados, donde los puntos de los datos se ponderan por los inversos de sus varianzas, de tal forma que las observaciones con varianzas grandes tienen menor impacto en el análisis que las observaciones asociadas a varianzas pequeñas. Este método permite corregir problemas de 
heterocedasticidad, produciendo estimadores lineales e insesgados.

El modelo de regresión lineal ponderado parte de considerar la regresión lineal que busca explicar la variación en la variable dependiente en respuesta a unos cambios suscitados en las variables independientes. La Ec.4 del modelo de regresión lineal es:

$$
\mathrm{Y}=x \beta+\varepsilon
$$

Donde:

$\mathrm{Y}=\left(\mathcal{y} 1+y_{2}+y_{3}+\ldots . . y_{n}\right)$ Es el vector de respuestas (aleatorias).

Donde el término Ynes el escalar correspondiente a la observación n-ésima de la variable endógena del modelo o variable dependiente. Esta variable tiene como dimensión (nx1) y por tanto constituye en un vector columna.

Por su parte, Xn es la observación n-ésima de la variable explicativa o independiente del modelo. Así, $x=\left(x 1+x 2+x 3 \ldots \ldots x_{\mathrm{n}}\right)$ es una matriz de tamaño $n * p$ que contiene ceros, unos o variables independientes y que recoge todas las observaciones de las variables exógenas del modelo.

Por su parte, $\beta=(\beta 1+\beta 2+\beta 3 \ldots \ldots \beta p)$ Es el vector de parámetros, los cuales indican el peso relativo de la variable en la ecuación y son desconocidos y por ende deben ser estimados por la ecuación lineal.

Finalmente, $\varepsilon=(\varepsilon 1+\varepsilon 2+\varepsilon 3 \ldots \ldots \varepsilon n)$ Es el vector de errores (aleatorios) donde se recoge que no logra ser explicado por medio de las variables independientes. Adicionalmente, puede recoger errores de especificación lineal y los posibles generados por la variable Yn.. En términos generales esta vector de errores estocásticos muestra en qué medida las variables del modelo tanto de tipo independiente como dependiente se dispersan de la regresión lineal.

Bajo este modelo se supone que el vector de errores $\varepsilon$ sigue una distribución normal con media igual a cero y matriz de varianza igual. Ahora suponiendo que se conocen las varianzas heterocedásticas $\sigma_{i}^{2}$, se divide a ambos lados de la Ec.(4) entre $\sigma_{i}$ se logra obtener las variables transformadas o estimadores MCP (mínimos cuadrados ponderados), y el término de error transformado $\varepsilon^{*}$, y que puede expresarse:

$$
\operatorname{var}\left(\varepsilon i^{*}\right)=1
$$

En este caso la varianza del término de error $\varepsilon^{*}$ * es ahora homocedástica.

Las variables incluidas en este estudio y que se pretenden explicar por medio de este método estadístico son: El componente de innovación, componente de inversión en capital humano y la variable ambiental.

Para obtener los estimadores de los parámetros desconocidos del modelo así como para realizar contrastes de hipótesis y la verificación del modelo, se necesitan un conjunto de hipótesis que, si bien se pueden ir desarrollando a medida que se vayan necesitando, se establecerán en esta sección, haciéndose referencia a ellas en el momento en el que se utilicen.

El conjunto de hipótesis sobre las que se basa el modelo de regresión versa sobre los siguientes aspectos:

1.- Forma funcional de la relación. (Supondremos que es lineal)

2.- Correcta especificación del modelo. (Es decir, que x es la única variable explicativa)

3.- La variable $\mathrm{x}$ es no estocástica.

4.- Identificabilidad de los parámetros. (b1 y b2 se podrán estimar de forma única)

5.- Valor esperado de la perturbación dada la información observada. $(\mathrm{E}(\varepsilon)=0(\mathrm{nx} 1))$

6.- Varianzas y covarianzas de las perturbaciones dada la información observada. $\mathrm{E}=\left(\varepsilon \varepsilon^{\prime}\right)=\sum \varepsilon$

\section{Resultados y discusión.}

Como resultado del análisis factorial, obtuvimos el componente de Innovación, bajo el método de componentes principales que permite agrupar aquellas variables que se relacionan con la 
posibilidad de realizar procesos de Innovación dentro de los subsectores colombianos. Dentro de este grupo se encuentran 7 variables, de las cuales 3 se relaciona con tipo de inversión al interior de los subsectores en innovación, 1 tienen que ver con procesos de asociación para la realización de innovación, 2 con instituciones de apoyo empresarial y 1 con tipo de protección a la innovación. Estas variables logran estar definidas por niveles relativamente altos de varianza explicada, especialmente las relacionas con tipo de inversión, que en todos los casos superan el 90\%. En orden descendente encontramos:

\section{Registro y aceptación de patentes}

2. Empresas con innovación organizacional

3. Empresas adecuadas tecnológicamente

4. Cooperación Empresas del grupo empresarial

\section{Empresas Incrementales}

6. Instrumentos para la Competitividad y el Desarrollo Productivo

7. Instrumentos de Educación nacional, formación profesional y para el trabajo

El segundo componente de estudio es el relacionado con Inversión en Capital Humano, donde la varianza logra ser explicada en un valor superior al $74 \%$. Dentro de este componente encontramos 4 variables relacionadas con niveles de educación formal, 2 variables que expresan niveles de inversión en capacitaciones como resultado de procesos de innovación y 1 variable que expresa niveles totales de inversión en formación para el desarrollo del capital humano.

\section{Postgrado}

2. Profesional

3. Inversión en capital Humano

4. Inversión en Capacitaciones relacionas con innovaciones organizacionales

\section{Doctorado}

6. Inversión en Capacitaciones relacionas con innovaciones

\section{Educación Intermedia}

Así pues se determina que en el caso del componente relacionado con la inversión en capital humano, la variable representativa es Postgrado debido a su alta explicación de su varianza dentro de este componente. Esta variable se mide por el número de empleados que poseen este nivel de educación en las empresas de los subsectores.

En el caso del componente de innovación, la variable utilizada es la Empresas incrementales, pues a pesar que no tiene la más alta puntuación en cargas, es la variable que puede recopilar la mayor cantidad de subsectores, y nos muestra un panorama actual de los niveles de inversión en innovación y por tanto esta variable recoge el efecto de las variables de su componente sobre la variable a explicar. A diferencia de Registro de patentes que solo agrupa a un porcentaje muy bajo, pero que logra tener una alta explicación de su varianza dentro del componente. La variable Empresas Incrementales se encuentra medida por el número de empresas de este tipo en cada subsector.

A partir de las variables obtenidas en el análisis factorial se ha realizado el análisis de regresión lineal ponderado. La variable dependiente a explicar es la productividad laboral por cada subsector colombiano, utilizando variables representativas para cada componente del análisis factorial, y una variable que recoge las capacidades ambientales y manejo de recursos, medida por el número de trabajadores de las empresas de los subsectores cuya labor está destinada a dicho fin. Las variables dependientes del modelo también se encuentran ponderadas por trabajador.

Los resultados del análisis de regresión son muy significativos. En este caso las variables que caracterizan los niveles de innovación, inversión en capital humano y el factor ambiental explican, aproximadamente, un $86 \%$ de la variabilidad de productividad laboral de los subsectores colombianos. El modelo presentado resulta admisible considerando que los valores de la $\mathrm{F}$ resultan muy significativos. 
Tabla 1. Resultados ANOVA

\begin{tabular}{clccccc}
\hline Modelo & & Suma de cuadrados & gl & Media cuadrática & F & Sig. \\
\hline \multirow{2}{*}{1} & Regresión & $3,341 \mathrm{E} 23$ & 3 & $1,114 \mathrm{E} 23$ & 52,113 &, $000^{\mathrm{a}}$ \\
& Residual & $1,197 \mathrm{E} 23$ & 56 & $2,137 \mathrm{E} 21$ & & \\
& Total & $4,538 \mathrm{E} 23$ & 59 & & & \\
\hline
\end{tabular}

a. Variables predictoras: (Constante), Emp Increm, Ambiental y manejo de residuos, Postgrado

b. Variable dependiente: VALOR AGREGADO

c. Regresión de mínimos cuadrados ponderados - Ponderada por Total

Tabla 2. Resultados Coeficientes

\begin{tabular}{|c|c|c|c|c|c|c|c|}
\hline \multirow[t]{2}{*}{ Modelo } & \multicolumn{2}{|c|}{ Coeficientes no estandarizados } & \multirow{2}{*}{$\begin{array}{c}\text { Coeficientes } \\
\text { tipificados } \\
\text { Beta }\end{array}$} & \multirow[t]{2}{*}{$\mathrm{t}$} & \multirow[t]{2}{*}{ Sig. } & \multicolumn{2}{|c|}{$\begin{array}{c}\text { Estadísticos de } \\
\text { colinealidad }\end{array}$} \\
\hline & $\mathrm{B}$ & Error típ. & & & & Tolerancia & FIV \\
\hline (Constante) & 246802180,932 & 112824830,061 & & 2,187 & 0,033 & & \\
\hline Postgrado & 1750713,550 & 343485,907 & 0,458 & 5,097 & 0,000 & 0,584 & 1,711 \\
\hline $\begin{array}{l}\text { Ambiental } \\
\text { y manejo de } \\
\text { residuos }\end{array}$ & 15944408,876 & 1958260,686 & 0,619 & 8,142 & 0,000 & 0,815 & 1,227 \\
\hline Emp Increm & $-10013790,430$ & 4082048,552 & $-0,202$ & $-2,453$ & 0,017 & 0,694 & 1,442 \\
\hline
\end{tabular}

En la Tabla No. 3 se aprecia que, el nivel de significancia del modelo es muy alto teniendo en cuenta que todos los factores como la inversión en capital humano y ambiental son significativos y afectan positivamente a la variabilidad productividad laboral de los subsectores colombianos.

Tabla 3. Resultados del Análisis de Regresión

\begin{tabular}{ccc}
\hline VARIABLES & $\beta$ & t-student \\
\hline Constante & $0,458^{*}$ & 2,187 \\
Postgrado $^{1}$ & $(-2,02)^{* *}$ & 5,097 \\
Empresas incrementales $^{2}$ & $0,619^{*}$ & $-2,453$ \\
Ambiental y recursos $^{2}$ & 8,142 \\
$\mathrm{R}^{2}$ & 0,736 \\
$\mathrm{~F}$ & 52,113 \\
Significación & 0,000 \\
\hline
\end{tabular}

* Nivel de significancia $\mathrm{p}<0,01$;

$* *$ Nivel de significancia $\mathrm{p}<0,05$

${ }^{1}$ Componente capital humano

${ }^{2}$ Componente de innovación

Por otra parte, los resultados del análisis de regresión destacan claramente una relación inversa de la innovación, representada por las Empresas Incrementales, con respecto a la productividad laboral. Este tipo de empresas se caracterizan por logra incorporar innovaciones pequeñas dentro del proceso productivo o a su producto final y por ende logra constituirse en un tipo de innovación frecuente dentro de los subsectores colombianos.

Este tipo de hallazgo no permite comprobar la hipótesis 2 de nuestro trabajo, debido a la relación negativa entre la variable representativa del factor innovación (Empresas incrementales) y la productividad laboral. Sin embargo, estos resultados confirman los hallazgos de otras investigaciones que demuestran que la innovación puede tener impacto negativo en la competitividad, si no logra desarrollar capacidades, que sean consecuencia de la combinación de las habilidades organizativas, funcionales y tecnológicas, así como los conocimientos y participación en redes de cooperación e involucrados en procesos de aprendizaje continuo, para realizar innovaciones constantes y habituales, generando una disminución en la rentabilidad inmediata y por ende impacta la productividad laboral como medida de la competitividad. (Teece, 1982; Galán, 1998; Díaz, 2003; Fernández, 2003;Lefebvre et al., 1998; Goñi, 2008; Merino y Moreno, 1996; Eusebio, 2004, Cavusgil, 
1984; Cooper y Kleinschmidt, 1985; Daniels y Robles, 1982; Aaby y Slater, 1989; Matthews citado por Escorza, 2001).

Por otra parte, el análisis destaca el efecto positivo de la formación sobre el nivel de productividad laboral permitiendo comprobar la hipótesis H1. El factor obtenido, dentro del análisis factorial, recoge el grado actual de formación de los trabajadores vinculados a los diferentes subsectores colombianos y la inversión asociada a procesos de capacitación.

En este caso, los resultados sugieren que a medida que la plantilla recibe una mayor formación, mejor será su contribución a la generación de valor de la organización y por ende a su subsector económico. Tal y como lo demuestran los resultados del análisis de regresión, la relación entre el mencionado factor y la productividad laboral de los subsectores colombianos es muy fuerte, estando en línea con los resultados obtenidos por Aguilar (2000), Sastre y Aguilar (2000), Casanova (2002), entre otros.

En cuanto a la variable relacionada con el manejo ambiental se tiene como resultado un impacto positivo en el modelo, es decir logra comprobar la hipótesis $\mathrm{H} 3$ planteada en este trabajo. Este resultado permite concluir que los sectores que contratan mayor cantidad de personas para el manejo ambiental mejoran sus resultados en la competitividad, pues es el capital humano el que realmente puede asumir los cambios que requiere este proceso y por ende mejorar la eficiencia empresarial. (Flórez y Jiménez, 2007; Millán y Nieto, 2003) y adicionalmente deja entrever los esfuerzos de la organización por mejorar y desarrollar recursos y capacidades, relacionados con el manejo ambiental, para el logro de la competitividad. (Rodríguez, 2007; Millán y Nieto, 2003).

\section{Conclusiones}

Este estudio presenta evidencia empírica que permite contribuir a entender mejor como los recursos y capacidades inciden en la productividad de los subsectores de una economía, generando externalidades para las empresas pertenecientes a él.
Se logró explicar bajo la teoría basada en recursos y capacidades que la consecución y sostenibilidad de la ventaja competitiva puede concebirse como el resultado de un proceso de acumulación de recursos unidos a procesos y rutinas organizativas, demostrando que los recursos de carácter intangible juegan un papel fundamental en la consecución y mantenimiento de la ventaja competitiva.

En concreto, este trabajo aporta información sobre la incidencia en el nivel de productividad dentro de los subsectores colombianos, de recursos y capacidades de tipo humano, ambiental einnovación. Este tipo de información permite que las empresas vinculadas a cada subsector, tomen decisiones sobre como logran desarrollar o mejorar los recursos y capacidades con los que cuentan y mejorar su desempeño e impulsar el de su subsector.

En este estudio podemos ver como los niveles de inversión en actividades de innovación tienden a ser muy reducidas lo cual afecta los niveles de competitividad. Es por eso que es necesario que se tome conciencia, desde el nivel empresarial, de generar innovaciones de tipo radical y no incremental para lograr que realmente se logren grandes cambios dentro de las organizaciones y se traslapen a los subsectores. Una solución para esta situación es por medio del desarrollo de capacidades bien sean de relación o de cooperación entre las empresas de un mismo sector o localización geográfica que permitan mejorar la trasferencia de conocimiento y apoyo reciproco, requerido para la generación de innovaciones de gran valor para el mercado. Con respecto, al desarrollo de recursos y capacidades ambientales a pesar de que en el corto plazo pueden generar costos a la organización ante su inversión, el no ser asumido por un productor o sector, limitan su posibilidad de llegar a ser competitivos. Sin embargo, algunos sectores pueden decidir no asumir dichos costos y obtener ventajas pero de carácter espurio e insostenible.

Se presenta evidencia de la necesidad de fortalecer los niveles de innovación en los diferentes sectores, pero especialmente en aquellos que son de clase mundial. 
Adicionalmente, se necesita la consolidación del aparato institucional o la creación de acuerdos región-región, ciudad-ciudad o sector-sector para impulsar el mejoramiento tecnológico y por ende industrial. Desde esta perspectiva, debe fomentarse el desarrollo de nuevos productos y procesos, aumentar las inversiones en investigación formal, mejorar la calidad de los productos actuales, entre otras acciones.

No obstante, es preciso consolidar nuevos programas para las empresas nacientes $\mathrm{y}$ fortalecer las existentes, para lo cual este trabajo brinda información sobre qué recursos y capacidades podrían articular sus estrategias de desarrollo empresarial que permita altos niveles de productividad. De igual forma, es necesaria la realización de acuerdos de colaboración como un medio importante para superar las deficiencias de recursos y capacidades y aumentar los niveles de productividad sectorial.

Asimismo, el factor ambiental podría llegar a analizarse de forma más exacta por medio de la inversión realizada, tecnología incorporada, efectos económicos entre otras. De igual forma, la innovación arrojaría grandes explicaciones si se contara con información relacionada con recursos financieros, expectativas de retorno, cultura organizacional, por dar algunos ejemplos.

\section{Referencias Bibliográficas}

Aaby, N.E. y Slater, S.T. (1989).Management Influences on Export Performance: A review of the Empirical Literature 1978-88, International Marketing Review 6, № 4, 7-23.

Aguilar, E.M. (2000). El diseño de la retribución de los directivos y su efecto sobre los resultados empresariales. Economía Industrial 333, 131148.

Barney, J.B. (1991). Firm Resources and sustained competitive advantage. Journal of Management 7

Bartmess, A. y Cerny, K. (1993). Building competitive advantage through a global network of capabilities. California Management Review 35, pp. 78-103.
Bell, M., \& Pavitt, K. (1992). National capacities for technological accumulation: Evidence and implication for developing countries. Paper prepared for the World Bank's. Washington.

Cantwell, J., \& Molero, J. (2003) Introduction, en J. Cantwell y J. Molero (comps.), Multinational Enterprises, Innovative Strategies and Systems of Innovation, Londres, Edward Elgar. Base de Datos SicenceDirect

Carlsson, B., Jacobsson, S., \& otros. (2002). Innovation systems: analytical and methodological issues. Research Policy 31, $\mathrm{N}^{\mathrm{o}}$ 2, Amsterdam, Elsevier.

Casanova, F. (2002). Formación desde los sindicatos. Experiencias innovadoras. Montevideo: Cinterfor/OIT, 2002.

Castellanos., Galves., \& otros. (2006). Medición Potencial de investigación y Desarrollo de las Pymes a partir de su Capacidad de Gestión Organizacional. Revista- Escuela de Administración de Negocios. Mayo- Agosto, Número 57.

Cavusgil. (1984). Differences among exporting firms based on their degree of Internationalization. Journal of Business Research 12, № 3, 195-208.

Cohen., \& Levinthal. (1989). Innovation and learning: The two faces of R\&D. The Economic Journal 99, September, 569-596.

Cohen., \& Levinthal. (1990). Absorptive capacity: A new perspective on learning and innovation. Administrative Science Quarterly 35, Issue 1, 128-152.

Cooke, P., Gómez, U. M., \& Etxbarria, G. (1997). Regional innovation systems: institutional and organisational dimensions, Research Policy 26, No 4-5, Amsterdam, Elsevier, diciembre.

Cooper, R., \& Kleinschmidt, E. (1985). The Impact of Export Strategy on Export Sales Performance. Journal of International Business Studies 16, No 1, 37-56.

Cruz, A. (2000). Protección ambiental, Desarrollo económico y competitividad. Desarrollo sustentable. México. 
Daniels, J., \& Robles, F. (1982). The choice of technology and export commitment: the peruvian textile industry. Journal of International Business Studies 13. Primavera/ Verano, 67-87.

Díaz, N.L. (2003). El conocimiento organizativo tecnológico y su incidencia en la innovación y performance corporativa. Evidencia para la empresa industrial española. Tesis doctoral. Universidad de Las Palmas de Gran Canaria.

Diéguez, M., Sinde, A. I., Gueimonde, E, A., (2007). Teoría de recursos y capacidades y teoría del capital humano: bases teóricas de la formación de recursos humanos como mecanismo de apoyo de las nuevas tecnologías de fabricación 43, $\mathrm{N}^{\circ}$ 253-254, p. 101-109.

Dosi, G. (1988). Sources, Procedures, and Microeconomics Effects of Innovation. JEL 26, N. 3, 1120-1171.

Duque, \& Tipia (2007). Componente costos ambientales para conducir negocios en Ecuador: Mejoramiento de la competitividad y protección del ambiente.

Edquist, C. (1998). Systems of innovation technologies, institutions and organizations. Science, Technology and The International Political Economy Series.

Erber, K., \& Gersten, K. (1997). Cooperation networks towards virtual enterprises. Virtualorganization. net, Newsletter 1 (5): 12-22.

Escorza, Pere., \& Valls, J. (2001). Tecnología e innovación en la empresa. España, Alfaomega, Ediciones UPC.

Espinosa, H. (2004) ¿Inversión en investigación y desarrollo para generar competitividad? Un análisis de efectos y determinantes a nivel de empresas manufactureras - Perú 1998

Eusebio, R. (2004). Innovación Tecnológica y Resultado Exportador: Un análisis empírico aplicado al sector textil-confección español. Revista Europea de Dirección y Economía de la Empresa 12, № 2, 73-88.
Fernández, M. (2003). Modelo de comportamiento de la organización virtual: Una aplicación empírica a los sistemas de franquicia. Tesis Doctoral Universidad de las Palmas de Gran Canaria.

Florez, P. \& Jimenez, S. (2007). Sistemas de Gestión Ambiental como Estrategia de Competitividad en la Industrial Química. Caso de Implantación: ColorQuimica S:A. Revista Producción + Limpia 2, $\mathrm{N}^{\circ} 2$. Diciembre.

Freeman. Ch. (1998) La economía del cambio tecnológico. Ariel, España.

Galán, J.L., \& Díez, E.C. (1998). Práctica de la franquicia. McGraw-Hill, Madrid

Geisse, G. (1990). Competitividad Económica y sustentabilidad ambiental. Diario El mercurio. Marzo.

Gill, I. (1989). Technological change, education and obsolescence of human capital: Some evidence for the U.S. Mimeo, noviembre.

Goñi. (2008). Talento Tecnología Y Tiempo. Ediciones Díaz De Santos, S.A., 2008

Grant, R.M. (1991). The resource-based theory of competitive advantage: Implications for strategy formulation, California Management Review 33

Hall, R. (1992). The strategic analysis of intangible resources. Strategic Management Journal 38, ํ 2, 135-144.

Hasenclever, L., \& Cassiolato, J. E. (1998). Capacitación Tecnológica Empresarial Brasileña y Transferencia de Tecnología. Revista de Economía y Empresa XII (II época, III cuatrimestre), 34, 15-31.

Howells, J. (1999). Regional systems of innovation?, en D. Archibugi, J. Howells y J. Michie, Innovation Policy in a Global Economy, Cambridge, Cambridge University Press.

Iammarino, S. (2005). An evolutionary integrated view of regional systems of 
innovation. Concepts, measures and historical perspectives. European Planning Studies 13, N ${ }^{\circ}$ 4, Londres, Taylor and Francis.

Koch, M.J., \& Macgrath, R.G. (1996). Improving labor productivity: Does Human Resource Management policies do matter. Strategic Management Journal 17, 5, 335-354.

Lefebvre, E., Lefebvre, L., \& Bourgault, M. (1998). R\&D-Related Capabilities as Determinants of Export Performace. Small Business Economics 10, 365-377.

Levitt, B., \& March, J.G. (1988). Organizational Learning. Annual Review of Sociology 14, 319340.

Malmberg, A., Sölvell, O. \& Zander, I. (1996). Spatial clistering, local accumulation of knowledge and firm competitiveness. Geografiska Annaler 78, № 2, Oxford, Reino Unido, Blackwell Publishing. Jstor

Martínez. (2001). Las nuevas tecnologías de la información y la comunicación en la educación superior. Extramuros. No. 14 (147-156).

Merino., \& Moreno, L. (1996). Actividad comercial en el exterior de las empresas manufactureras españolas, y estrategias de diferenciación de producto. Papeles de Economía Española, № 66, 107-123.

Mertens, L. (2002). Formación, productividad y competencia laboral en las organizaciones: conceptos, metodologías y experiencias. Montevideo: Cinterfor. 179.

Millan, P., \& Nieto, L. M. (2003). Ingeniería y Gestión Ambiental: Una propuesta Practica de Formación Científica, Técnica y Ética. Articulo resultante del XIX Congreso Nacional de la AMIDIQ. Mayo.

Nelson, R. R., \& WINTER, S. G. (1992) Evolutionary Theory of Economic Change. Cambridge, Massachusetts London: The Belknap Press of Harvard University Press.

Nelson, R., \& Phelps, E. (1996). Investments in Humans, Technological Diffusion and
Economic Growth", American Economic Review, 61, p. 139-162.

Parizotto, J. A. O. (1995). Gerenciamiento Ambiental: Estudio del Caso de Cinco Empresas de Minerales en Brasil. Rio de Janeiro, CNPq/ CETEM,. (Série Qualidade e Produtividade, 5).

Paunero, X. (2003). Innovación Empresarial y territorio. El SAL - Sistema Agroindustrial localizado en el noreste de Cataluña". Retour au Menu.

Pitt, J. C. (2000). Thinking About Technology: Foundations of the Philosophy of Technology. Seven Bridges Press, New York.

Porter, M. \& McGahan, A. (1997). How Much Does Industry Matter, Really?. Strategic Management Journal 18, July, 1997: 15-30.

PORTER, M., \& Van Der Linde, C. (1995). Toward a new conception of the environment - competitiveness relationship. Journal of Economic Perspectives 9, № 4.

Puig, I., Freire, J., \& Almazor, L. (2007). Efectos de la política Ambiental sobre la competitividad. Revista Iberoamericana de Economía Ecológica 6, 52- 61.

Robledo y Ceballos. (2008). Estudio de un proceso de innovación utilizando la dinámica de sistemas. Cuadernos de Administración 35 N. 1, Bogotá, enero - junio. 2008

Rodeiro, D., \& López, M. (2007). La innovación como factor clave en la competitividad empresarial: un estudio empírico en pymes. Revista Galega de Economía 16, №2.

Rodríguez. (2007). Gestión ambiental municipal y participación local. Decimo tercer informe sobre el estado de la Nación en desarrollo humano sostenible.

Sastre, M.A.; \& Aguilar, E.M. (2000). Un modelo de competitividad empresarial basado en los recursos humanos. Economía Industrial 332, 125-138. 
Schmalensee, R.. (1994). The Costs of Environmental Protection. En M. B Kotowski. (ed.). Balancing economic growth and environmental goals. Washington, DC: American Council for Capital Formation Center for Policy Research.

Sisson, K., \& Store, J. (2002) Realities of Human Resource Management: Managing the Employment Relationship", Buckingham, Open University Press.

Snell, S., \& Dean, J. (1992). Integrated Manufacturing and Human Resource Management: A Human Capital Perspective. Academy of Management Journal 35, $\mathrm{N}^{\mathrm{o}} 3$, 467-504.
Taylor, J.R. (1993). Rethinking the theory of organizational communication: How to read an organization. Ablex Publishing, Norwood.

Teece, D. (1982). Towards an Economic Theory of The Multiproduct Firm. En Journal of Economic Behavior and Organization, $\mathrm{N}^{\circ} 3$.

Teece, D., \& Pizano, G. (1994). The dynamic capability of firms: An introduction. Industrial and Corporate Change 3 (3), 537-556

Uribe, E. (2007). Reflexiones sobre el impacto social de la innovación empresarial. CONCYTEG. $\mathrm{N}^{\circ} 28$, Noviembre.

Williams, E., Macdonald, K., \& Kind, V. (2002). Unraveling the competitiveness debate. European Environment 12, No. 5, 284-290. 